\title{
Anterior knee pain
}

\author{
Jung Eun Lee • Kyung Nam Ryu • Ji Seon Park • \\ Chung Soo Han • Yong Koo Park
}

Published online: 12 December 2014

(C) ISS 2014

\section{Discussion}

Radiographs of the right knee (Fig. 1) showed a superolateral bipartite patella. An osteolytic lesion with lobulated margins involved the accessory fragment of the bipartite patella and extended through the adjacent fibrocartilaginous zone and involved the patella. CT (Fig. 2) revealed the osteolytic lesion to have a well-defined thin sclerotic margin with small internal calcific foci. MR imaging (Fig. 3) of the lesion revealed low signal intensity on both T1- and T2-weighted images. Most of the lesion enhanced following contrast administration, sparing the central portion. With the exception of mild adjacent soft tissue perilesional enhancement, there was no evidence of synovial thickening or periarticular inflammation.

On arthroscopy, the fibrocartilaginous zone between the patella and accessory fragment was disrupted by fibrous tissue. Following incision of the fibrous tissue, a large amount of

The case presentation can be found at doi: 10.1007/s00256-014-2069-1.

J. E. Lee $\cdot$ K. N. Ryu $(\bowtie) \cdot J$. S. Park

Department of Radiology, Kyung Hee University Hospital, 1, Hoegi-dong, Dongdaemun-ku, Seoul 130-702, Republic of Korea e-mail: t2star@khu.ac.kr

\section{S. Han}

Department of Orthopedic Surgery, Kyung Hee University Hospital,

1, Hoegi-dong, Dongdaemun-ku, Seoul 130-702, South Korea

\section{Y. K. Park}

Department of Pathology, Kyung Hee University Hospital, 1, Hoegi-dong, Dongdaemun-ku, Seoul 130-702, Korea

tophaceous material flowed freely from the lesion. Histologic examination of this material revealed negatively birefringent, needle-shaped, crystal deposits surrounded by fibrotic tissue and zones of polymorphic foreign-body reaction with multinuclear giant cells confirming the diagnosis of tophaceous gout (Fig. 4).

Since the first report of gout in the patella by Peloquin and Graham [1] in 1955, there have been only a few reports of tophaceous gout developing in the patella. Gout arising in a bipartite patella was first reported by Reber et al. [2] in 1996, describing findings similar to this report with the lesion involving the patella, the accessory fragment and traversing the intervening fibrocartilaginous zone [2,3]. Walot and Staple [4] speculated that prior trauma could be a causative factor for the development of tophaceous gout in the patellar or bipartite patella. Cohn et al. [5] speculated that the area of pseudarthrosis of the bipartite joint could change the phagocytotic capability of insoluble urate crystals, leading to the development of gouty arthritis. On the basis of arthroscopic observations of tophaceous gout in a patient with a bipartite patella, Kobayashi et al. [6] found that urate precipitation was surrounded by an inflammatory synovial sheath and speculated that some pre-existing inflammatory condition around the accessory fragment induced deposition of urate crystals, and destruction of the barrier between the urate deposits and the joint causing an acute arthritis.

In the present case, the patient did not have a history of trauma. However, the knee was repetitively stressed from activities such as mountain climbing and bicycle riding. We speculate that this could have led to the formation of an abnormal pseudarthrosis in the bipartite joint with chemical and biological changes within this joint creating an environment suitable for urate precipitation and subsequent bone involvement.

It is not easy to make the diagnosis of tophaceous gout in cases of a solitary intraosseous patella lesion. Although they 
Fig. 1 Anteroposterior (A) and skyline view (B) radiographs of the right knee show an osteolytic lesion with lobulated margins in the superolateral portion of the patella. It is underlying a superolateral bipartite patella
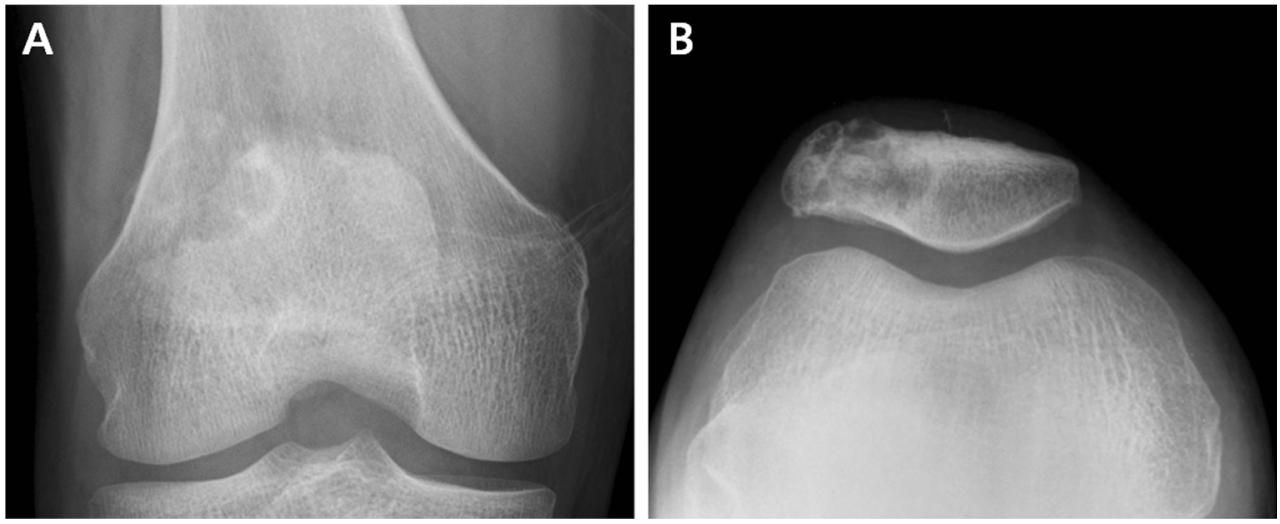

Fig. 2 Coronal (A) and axial (B) computed tomography (CT) images of the right knee show a well-defined osteolytic lesion with a thin sclerotic rim. Calcific mineralization within the lesion (white arrows), including the fibrocartilaginous zone, is identified
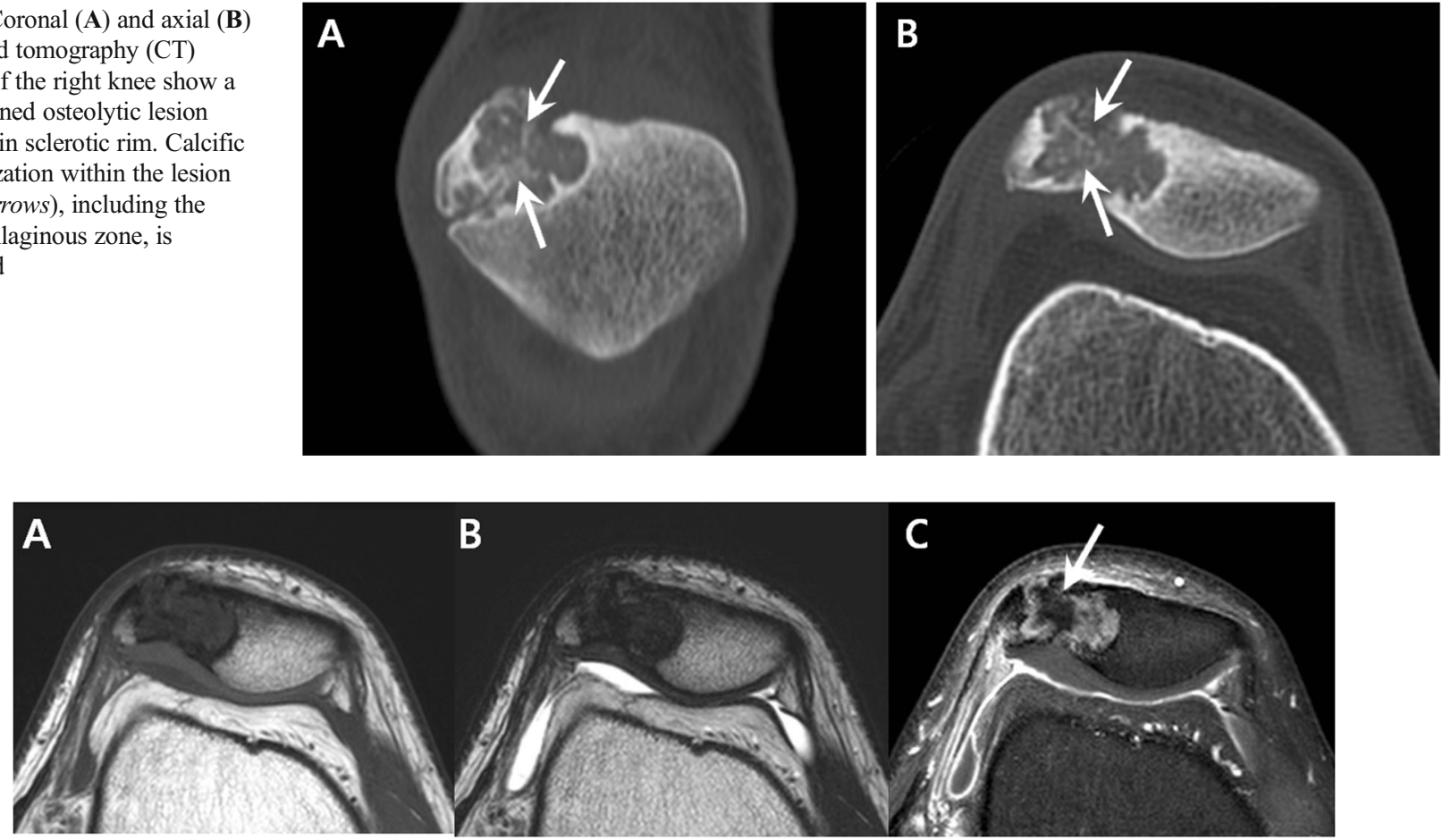

Fig. 3 Magnetic resonance (MR) images show a lesion with low signal intensity on T1- (TR/TE, 400/20) (A) and T2-weighted (TR/RE, 1950/ 100) (B) images. Most of the area of the lesion was enhanced on the

contrast enhanced T1-weighted fat-suppression image (TR/TE, 466/20) (C), except for the central portion (white arrow)

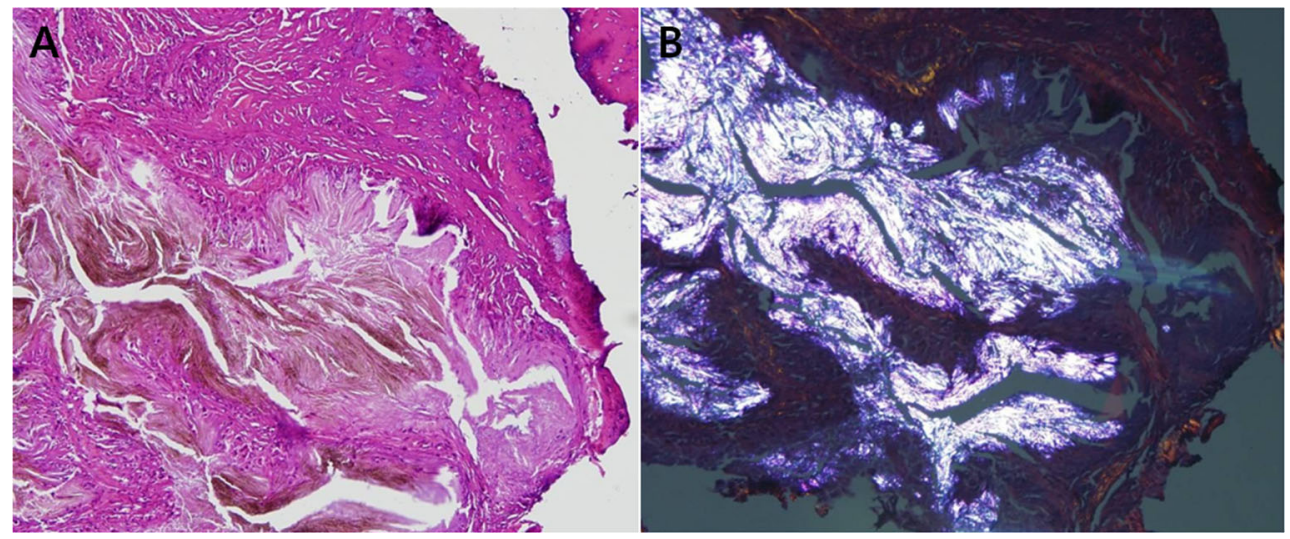

Fig. 4 (A) Histological appearance of a gouty tophus with its typical aggregate of needle shaped monosodium uric acid crystals surrounded by a foreign body reaction and chronic inflammation $(\mathrm{H} \& \mathrm{E}, \mathrm{x} 400)$. (B) There are needle-shaped crystals on polarized optical microscopy 
are uncommon, several tumors of the patella should be considered in the differential diagnosis such as a giant cell tumor, chondroblastoma, osteoblastoma, hemangioma, hemangioendothelioma, osteosarcoma, and metastases [4, $7,8]$. In cases with evident internal mineralization, as seen in this case, the differential diagnosis must also include a chondroid tumor.

In the present case, however, considering the patient's age and imaging findings, the differential diagnosis can be narrowed. The appearance of the indolent activity suggested by the sclerotic margin would be quite atypical for an osteosarcoma or other malignant tumors. Chondroblastoma can rarely occur in older adults and usually have adjacent reactive marrow and soft tissue changes. Similarly, the matrix and sclerotic margin would make a giant cell tumor quite unlikely.

Recht et al. [9] reported that the possibility of gout should be considered when a geographic osteolytic lesion with a peripatellar calcific soft tissue mass is seen at the superolateral portion of the patella. CT is helpful for detecting mineralization and cortical destruction. MR features of gouty tophi are nonspecific with low-to-intermediate signal intensity on T1weighted images and variable, but commonly, low-tointermediate signal intensity on T2-weighted images, with the decreased signal due to calcium, crystal, or fibrous tissue. Synovial thickening with hypervascularity may be helpful for the diagnosis, but is not specific.

In conclusion, we describe tophaceous gout involving the accessory bone of a bipartite patella with extension to the adjacent fibrocartilaginous zone and patella. Although tumorous lesions developing in the patella should be considered in the differential diagnosis, knowledge of the features that characterize gout, such as a superolateral location, internal mineralization, adjacent bony erosion, or suggestive MR features, may lead to the appropriate diagnosis and treatment of the lesion.

\section{References}

1. Peloquin LU, Graham JH. Gout of the patella; report of a case. N Engl J Med. 1955;253(22):979-80.

2. Reber P, Crevoisier X, Noesberger B. Unusual localisation of tophaceous gout. A report of four cases and review of the literature. Arch Orthop Trauma Surg. 1996;115(5):297-9.

3. Enomoto H, Nagosi N, Okada E, Ota N, Iwabu S, Kamiishi S. Hemilaterally symptomatic bipartite patella associated with bone erosions arising from a gouty tophus: a case report. Knee. 2006;13(6): 474-7.

4. Walot I, Staple TW. Case report 539. Tophaceous gout of the patella. Skeletal Radiol. 1989;18(3):233-6.

5. Cohn BTIJ, Jackson DW. Erosion of the patella end in gout. Am J Sports Med. 1988;16:421-3.

6. Kobayashi KDM, Okuhara A, Adachi N, Yasumoto M, Ochi M. Tophacenous gout in the bipartite patella with intraosseous and intraarticular lesions: a case report. J Orthop Surg (Hong Kong). 2005;13(2):199-202.

7. Casadei R, Kreshak J, Rinaldi R, Rimondi E, Bianchi G, Alberghini M, et al. Imaging tumors of the patella. Eur J Radiol. 2013;82(12):2140-8.

8. Singh J, James SL, Kroon HM, Woertler K, Anderson SE, Davies AM. Tumour and tumour-like lesions of the patella-a multicentre experience. Eur Radiol. 2009;19(3):701-12.

9. Recht MP, Seragini F, Kramer J, Dalinka MK, Hurtgen K, Resnick D. Isolated or dominant lesions of the patella in gout: a report of seven patients. Skeletal Radiol. 1994;23(2):113-6. 